\title{
Increased platelet distribution width and reduced IL-2 and IL-12 are associated with thrombocytopenia in Plasmodium vivax malaria
}

\author{
Allyson Guimarães Costa ${ }^{1,2,3,4,5}$, Yury Oliveira Chaves ${ }^{6,7}$, Andréa Teixeira-Carvalho ${ }^{8}$, \\ Rajendranath Ramasawmy ${ }^{1,2,5,9}$, Lis Ribeiro Valle Antonelli ${ }^{10}$, Lucas Barbosa ${ }^{6}$, \\ Antonio Balieiro ${ }^{6}$, Wuelton Marcelo Monteiro ${ }^{1,2}$, Maria Paula Mourão, ${ }^{1,2}$, \\ Marcus Vinicius Guimarães Lacerda ${ }^{1,2,4,6}$, Olindo Assis Martins-Filho ${ }^{3,8}$, \\ Fabio Trindade Maranhão Costa ${ }^{11}$, Adriana Malheiro ${ }^{1,3,4,5}$, Paulo Afonso Nogueira ${ }^{2,5,6 /+}$
}

${ }^{1}$ Universidade do Estado do Amazonas, Programa de Pós-Graduação em Medicina Tropical, Manaus, AM, Brasil

${ }^{2}$ Fundação de Medicina Tropical Dr Heitor Vieira Dourado, Instituto de Pesquisa Clínica Carlos Borborema, Manaus, AM, Brasil

${ }^{3}$ Fundação Hospitalar de Hematologia e Hemoterapia do Amazonas, Diretoria de Ensino e Pesquisa, Manaus, AM, Brasil

${ }^{4}$ Universidade do Estado do Amazonas, Programa de Pós-Graduação em Ciências Aplicadas à Hematologia, Manaus, AM, Brasil

${ }^{5}$ Universidade Federal do Amazonas, Programa de Pós-Graduação em Imunologia Básica e Aplicada, Manaus, AM, Brasil

${ }^{6}$ Fundação Oswaldo Cruz-Fiocruz, Instituto Leônidas e Maria Deane, Programa de Pós-Graduação em Biologia da Relação Patógeno-Hospedeiro, Manaus, AM, Brasil

${ }^{7}$ Fundação Oswaldo Cruz-Fiocruz, Instituto Oswaldo Cruz, Programa de Pós-Graduação em Biologia Parasitária, Rio de Janeiro, RJ, Brasil

${ }^{8}$ Fundação Oswaldo Cruz-Fiocruz, Centro de Pesquisas René Rachou, Grupo Integrado de Pesquisas em Biomarcadores, Belo Horizonte,

MG, Brasil

${ }^{9}$ Universidade Nilton Lins, Faculdade de Medicina, Manaus, AM, Brasil

${ }^{10}$ Fundação Oswaldo Cruz-Fiocruz, Instituto René Rachou, Laboratório de Biologia e Imunologia de Doenças Infecciosas e Parasitárias, Belo Horizonte, MG, Brasil

${ }^{11}$ Universidade Estadual de Campinas, Instituto de Biologia, Campinas, SP, Brasil

BACKGROUND Thrombocytopenia in malaria involves platelet destruction and consumption; however, the cellular response underlying this phenomenon has still not been elucidated.

OBJECTIVE To find associations between platelet indices and unbalanced Th1/Th2/Th17 cytokines as a response to thrombocytopenia in Plasmodium vivax infected (Pv-MAL) patients.

METHODS Platelet counts and quantification of Th1/Th2/Th17 cytokine levels were compared in 77 patients with uncomplicated $P$. vivax malaria and 37 healthy donors from the same area (endemic control group - ENCG).

FINDINGS Thrombocytopenia was the main manifestation in 55 patients, but was not associated with parasitaemia. The Pv-MAL patients showed increases in the mean platelet volume (MPV), which may be consistent with larger or megaplatelets. Contrary to the findings regarding the endemic control group, MPV and platelet distribution width (PDW) did not show an inverse correlation, due the increase in the heterogeneity of platelet width. In addition, the Pv-MAL patients presented increased IL- $1 \beta$ and reduced IL-12p70 and IL-2 serum concentrations. Furthermore, the reduction of these cytokines was associated with PDW values.

MAIN CONCLUSIONS Our data demonstrate that an increase in MPV and the association between reductions of IL-2 and IL-12 and PDW values may be an immune response to thrombocytopenia in uncomplicated $P$. vivax malaria.

Key words: platelet - Plasmodium vivax - IL-12p70 - IL-2 - IL-1 beta - thrombocytopenia

Thrombocytopenia is one of the most frequent haematological alterations in acute malaria infections involving Plasmodium falciparum and Plasmodium vivax malaria (PV-MAL). ${ }^{(1)}$ Thrombocytopenia is prominent in patients with splenomegaly and can be found in patients

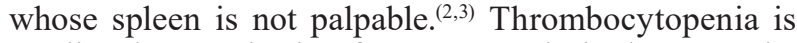
not listed as a criterion for severe malaria, however, its clinical importance has been widely recognised when accompanied by multiple organ failures. ${ }^{(4,5,6)}$ The mortality rate in vivax malaria patients with severe thrombo-

doi: 10.1590/0074-02760200080

Financial support: FAPEAM (Pró-Estado Program - \#002/2008 and PAPAC Program - \#005/2019), CNPq, CAPES.

WM, ML, FTMC, AM and PAN are research fellows from CNPq.

+ Corresponding author: paulo.nogueira@fiocruz.br

(D) https://orcid.org/ 0000-0003-3015-1021

Received 23 February 2020

Accepted 26 June 2020 cytopenia alone can be comparable to that of falciparum malaria when associated with other danger signs of malaria (such as seizures, jaundice, bleeding, among others), and these can serve as red flags in order to promptly identify patients who may have severe malaria. . $^{(4,6,7,8)}$

The pathogenesis of thrombocytopenia caused by malaria involves multifactorial phenomenon and leads to destruction and consumption of platelets. ${ }^{(9)}$ In particular, in falciparum malaria, platelets also bind diffusely in systemic microvasculature, rather than pooling in the liver or spleen. ${ }^{(10,11)}$ Moreover, other pathophysiological mechanisms have recently been associated with thrombocytopenia caused by malaria, such as coagulopathy, splenic sequestration of damaged platelets, platelet aggregation, antibody-mediated platelet destruction and oxidative stress aggregation formation and dysmegakaryopoiesis. $(4,12,13)$ In PV-MAL, thrombocytopenia is also common, however its pathogenesis is less well-known. 
Platelets are small disc-shaped cellular fragments with no nucleus, and which have regenerated continuously from megakaryocyte (MK). The MKs are large, polyploid cells that have lost their proliferative ability and have become progressively differentiated, creating an invaginated membrane system for platelet formation. (14) The steady state megakaryopoiesis is responsible for releasing of approximately $100 \times 10^{9}$ platelets per day and lifespan is 7-10 days. Under inflammatory and thrombocytopenia conditions, such as malarial infection, platelet lifespan is reduced to 2-3 days and MKs differentiate in order to replenish platelets. ${ }^{(15,16)}$

In general, thrombocytopenia in malaria is accompanied by both higher mean platelet volume (MPV) and larger platelet distribution width (PDW), which arise concomitantly with reduced platelet counts. ${ }^{(12,17)}$ The increase in platelet volume is a result of the release of larger platelets in the circulation, also described as giant or mega platelets. ${ }^{(4,13,18,19,20)}$ Mega platelets are larger than $5 \mu \mathrm{m}$ in the smear and are found in the peripheral circulation when there is increase of megakaryocyte production, due to reduced platelet level in the body. Even if the presence of mega platelets is not identified in the smear, it is possible to detect the increase in platelet volume indirectly, by increasing the MPV. Mega platelets also have a greater amount of granules, which, consequently, increases their ability to adhere and aggregate ${ }^{(19)}$ Mega platelets and increased MPV may indicate a compensatory premature release of platelets from the bone marrow in order to compensate for the lower absolute number of platelets in the periphery. ${ }^{(4,18,19,20)}$ According to other studies, the megakaryocytic lineage is apparently preserved in bone marrow and able to release mega platelets. ${ }^{(4,13,18)}$ Since the blood stream medullary response can be triggered by cytokines, our study assessed serum concentrations of $\mathrm{Th} 1 / \mathrm{Th} 2 / \mathrm{Th} 17$ cytokines, in order to characterise a profile of the immune response in thrombocytopenia caused by $P$. vivax infections.

\section{SUBJECTS AND METHODS}

Study area and population - $77 \mathrm{Pv}-\mathrm{MAL}$ patients (diagnosed as confirmed cases via examination of the thick blood smear) were enrolled at the Tropical Medicine Institute in the municipality of Coari, Amazonas State, Brazil. Coari is located at latitude: -4.08488 , Lon-

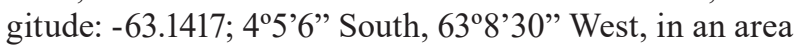
with a high incidence of malaria. It is estimated that approximately $30 \%$ of its inhabitants are at risk of malaria (annual parasitological index $>50$ ). P. vivax is responsible for $99.6 \%$ of cases of malaria. ${ }^{(21)}$ All the patients had classic malaria symptoms with no signs of severe or complicated malaria.

Parasitaemia levels were defined as low parasitaemia $\left(\leq 500\right.$ parasites $\left./ \mathrm{mm}^{3}\right)$ or mild $\left(>500\right.$ parasites $\left./ \mathrm{mm}^{3}\right)$. Blood cells were examined using an ABX Micros 60 haematology analyser (Horiba). 37 healthy donors were included in the study to form an endemic control group (ENCG). These were health professionals who are residents of this area, who had no previous history of malaria and tested negative in two thick blood smear examina- tions which were performed at intervals of 15 days. When detected, thrombocytopenia was classified as being either mild $\left(50-150,000 / \mathrm{mm}^{3}\right)$ or severe $\left(<50,000 / \mathrm{mm}^{3}\right) \cdot{ }^{(22)}$

A detailed serological screening was performed for hepatitis B virus, hepatitis C virus and HIV (Arquitect i2000SR, Abbott Diagnostics), and supervised by the Blood Bank (HEMOAM). In addition, participants were also screened for dengue Virus by polymerase chain reaction (PCR). After malaria diagnosis, blood samples were collected in EDTA tubes (BD Biosciences, USA), and sent in thermal boxes (maintained at $4^{\circ} \mathrm{C}$ ) to the Molecular Biology Laboratory of the Federal University of Amazonas in Coari.

Serum cytokine measurements - Frozen plasma samples were sent on dry ice to the Core Flow Cytometry Facility at the HEMOAM blood bank for cytokine measurement with the cocktail kit (CBA-BD/Biosciences Pharmingen, USA). The cytokines IL-2, IL-4, IL5, IL-6, IL-10, TNF, IFN- $\gamma$ and IL-17A were measured according to manufacturer's instructions, and IL-1 $\beta$, IL-8 (CXCL-8) and IL-12 (IL-12p70) were measured by ELISA (BD OptEIA Set II human kit, BD Biosciences Pharmingen, USA).

Statistical analysis and data presentation - All data were analysed using GraphPad prism software version 5. The Th1/Th2/Th17 cytokines levels were separated into non-thrombocytopenic, mild and severe thrombocytopenia according to platelet counts. Reference intervals for the platelet parameters according to the recommended European Federation of Clinical Chemistry and Laboratory Medicine were used.(23) In both analyses, groups were compared with the endemic control group, which represented the basal state of cytokine levels. The Mann Whitney test was used to compare platelet counts and cytokine levels of Pv-MAL patients with those of the ENCG. In addition, platelet counts were compared among patients of the malaria group and classified into low and mild parasitaemia. Spearman's rank correlation coefficient was used to assess the relationship between MPV and PDW indexes with platelet counts for the malaria group and endemic control group. A multinomial model classified the assessed platelet indices (Platelet counts, MPV and PDW indices) and cytokines between patients of the malaria group and classified them as having either low or mild parasitaemia. Finally, linear regression was performed to assess the predictors of thrombocytopenia in $\mathrm{Pv}$-MAL patients in relation to the ENCG, taking into account platelet count indices and cytokine serum concentrations. An asterisk (*) indicates a significance level of $\mathrm{p}<0.05,(* *) \mathrm{p}<0.005$ and $(* * *) \mathrm{p}<0.0005$.

Ethics - All protocols and consent forms were approved by the Research Ethics Committee at HEMOAM, under approval No. \#449.864/2011. Participants read and signed the written informed consent form prior to enrollment, according to Declaration of Helsinki and Resolution 466/12 of the National Health Council for research involving human subjects. All patients were treated according to recommendations of Brazilian Ministry of Health. 


\section{RESULTS}

All patients included in the study had uncomplicated paroxysms at the time of seeking medical care. None of the patients reported diarrhea or vomiting. $53(68.8 \%)$ patients were male and $24(31.2 \%)$ were female. 15 of the patients $(19.5 \%)$ reported primary malaria and 62 reported at least one previous malaria infection. None of the patients showed signs of anaemia or severe malaria. 30 patients had leukopenia (38.9\%) and lymphopenia was evident in 32 patients $(41.5 \%)$.

Thrombocytopenia was observed in 55 of 77 patients (71.4\%), also in five of the 37 individuals in the ENCG. Thrombocytopenia was classified as being either moderate $\left(51-149 \times 10^{3}\right.$ platelets $\left./ \mu \mathrm{L}\right)$ or severe (below $50 \times 10^{3}$ platelets $/ \mu \mathrm{L})$. Among the patients, $44(80 \%)$ had moderate thrombocytopenia and 11 displayed severe thrombocytopenia (20\%); none of the patients needed platelet transfusion. In Fig. 1A, coloured dots illustrate the PvMAL patients classified into non-thrombocytopenic, mild and severe thrombocytopenic individuals. The average age of the patients was $37.0 \pm 14.3$ years, the age of Pv-MAL patients did not affect platelet count according to Spearman's rank correlation coefficient of 0.1373 (p- value $=0.2338$ ). Among the 37 individuals used as the ENCG, three had moderate thrombocytopenia (8.1\%) and two (5.4\%) had severe thrombocytopenia and coloured as thrombocytopenia status. The average age of the patients was $29.4 \pm 8.45$ years in ENCG, though this did not influence the platelet count $(\mathrm{r}=0.2365$ and $\mathrm{p}$-value $=0.0738)$.

Platelet counts were used to assess whether the thrombocytopenia caused by vivax malaria was due to peripheral destruction or bone marrow disease. The average MPV index in Pv-MAL patients was higher than the average in the ENCG, although both were within normal range (Fig. 1B), and PDW values did not differ among the ENCG (Fig. 1C). No patient presented high parasitaemia. 38 patients (49.4\%) had low parasitaemia, with parasite counts being below 500 parasites per milliliter $\left(\leq 500\right.$ parasites $\left./ \mathrm{mm}^{3}\right)$, while $39 \mathrm{Pv}$-MAL patients $(50.6 \%)$ showed moderate parasitaemia (between 501 to 20,000 parasites $/ \mathrm{mm}^{3}$ ). However, not all platelet indices differed in relation to parasitaemia (Fig. 2A-C).

In general, lower platelet counts are associated with higher platelet volumes. Both groups showed an inverse correlation between mean platelet volume (MPV) and platelet counts (Fig. 3A-D), though not in relation to PDW
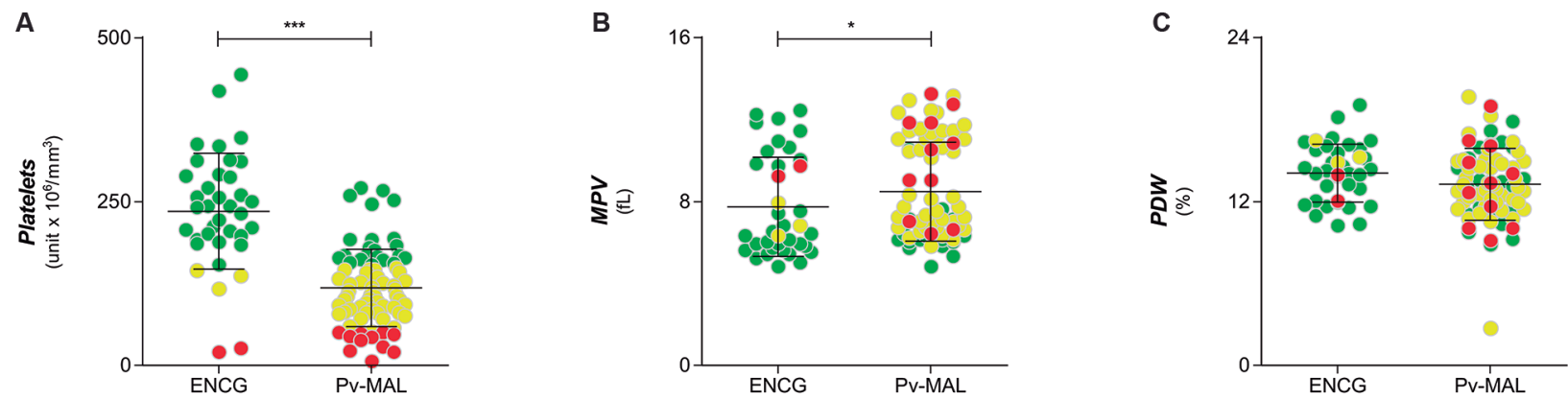

Fig. 1: comparison between platelet counts in Plasmodium vivax malaria (Pv-MAL) patients and endemic control group (ENCG). (A) Comparison of platelet counts in ENCG and Pv-MAL patients. Thrombocytopenia was defined as a platelet count less than $150,000 / \mathrm{mm}^{3}$ and then classified as mild $\left(50-150,000 / \mathrm{mm}^{3}\right)$ and severe $\left(<50,000 / \mathrm{mm}^{3}\right)$ thrombocytopenia. Patients were classified as non-thrombocytopenic when they had platelet counts above $150,000 / \mathrm{mm}^{3}$. The individuals were grouped according in non-thrombocytopenic (green circle), mild (yellow circle) and severe thrombocytopenia (red circle). (B) Comparison of mean platelet volume (MPV) in Pv-MAL patients and ENCG; (C) Comparison of platelet distribution width (PDW) in Pv-MAL patients and ENCG.
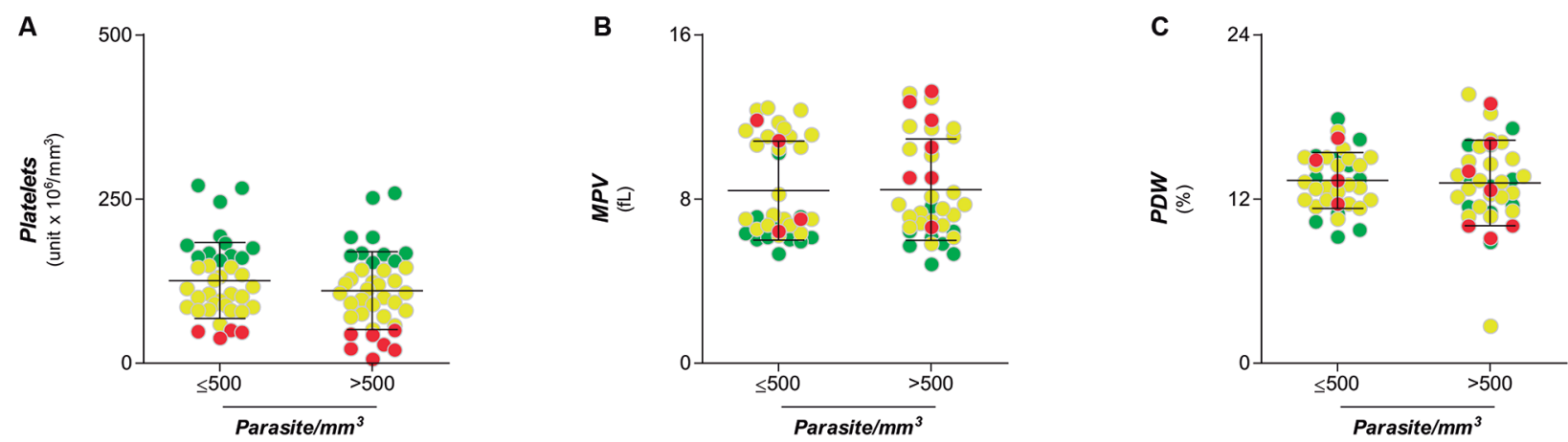

Fig. 2: comparison of parasitaemia with platelet indices. (A) Comparison of platelet indices according to parasitaemia classified in $\leq 500$ parasites $/ \mathrm{mm}^{3}$ and 501-to-20,000 parasites $/ \mathrm{mm}^{3}$. (B) Comparison of mean platelet volume (MPV) in relation to parasitaemia; (C) Comparison of platelet size heterogeneity (platelet distribution width - PDW) in relation to parasitaemia. The individuals were grouped according in nonthrombocytopenic (green circle), mild (yellow circle) and severe thrombocytopenia (red circle). 

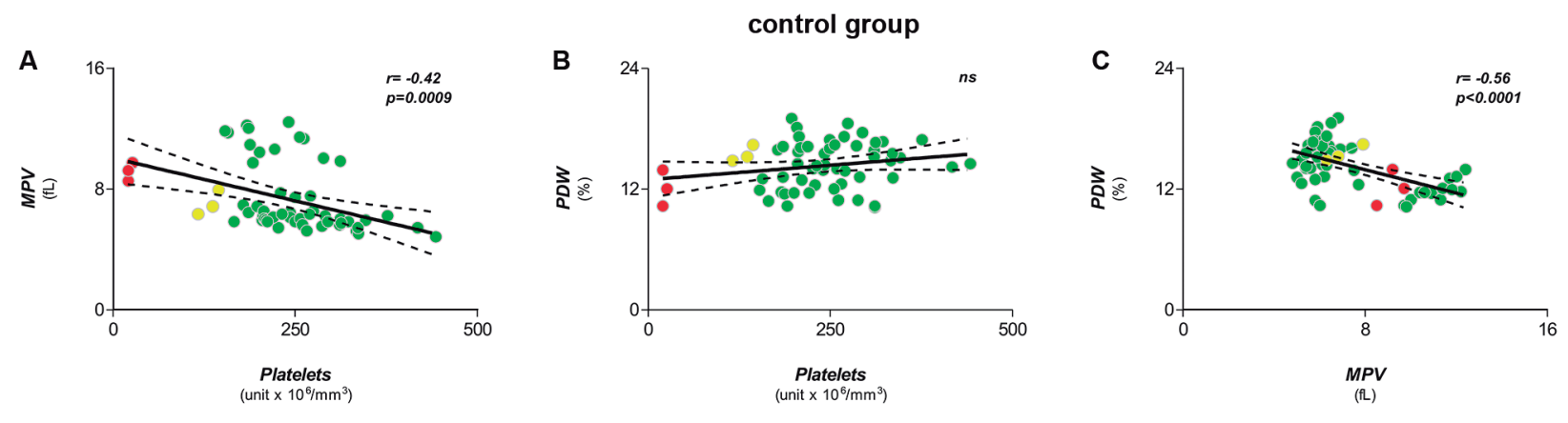

malaria group
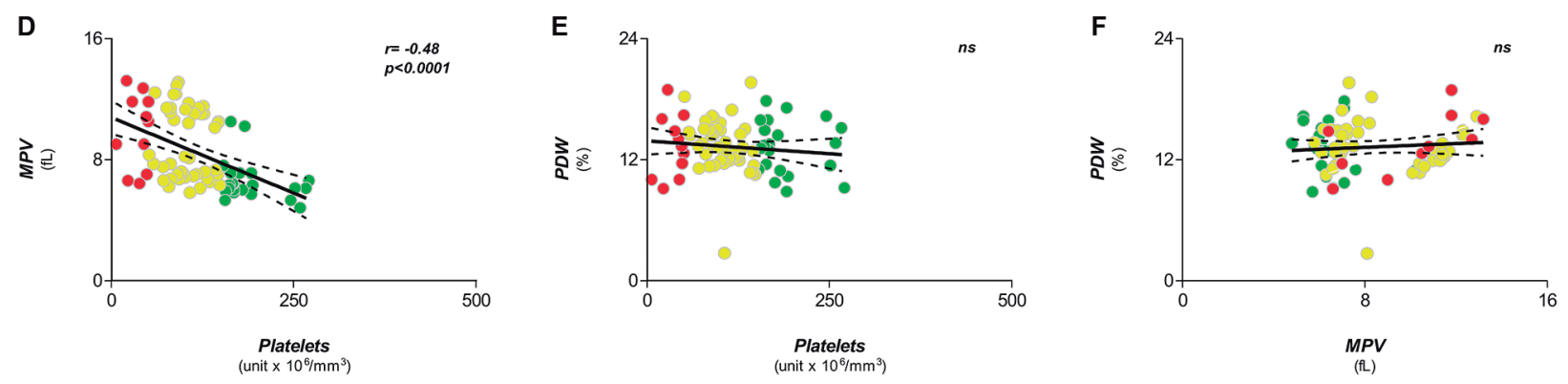

Fig. 3: comparison of correlations among platelet indices between Plasmodium vivax malaria (Pv-MAL) patients and endemic control group (ENCG). (A-C) Correlations between mean platelet volume (MPV) and platelet counts, platelet distribution width (PDW) and platelet counts, PDW and MPV in ENCG. (D-F) Correlations between MPV and platelet counts, PDW and Platelet counts, PDW and MPV in PV-MAL. The individuals were grouped according in non-thrombocytopenic (green circle), mild (yellow circle) and severe thrombocytopenia (red circle).
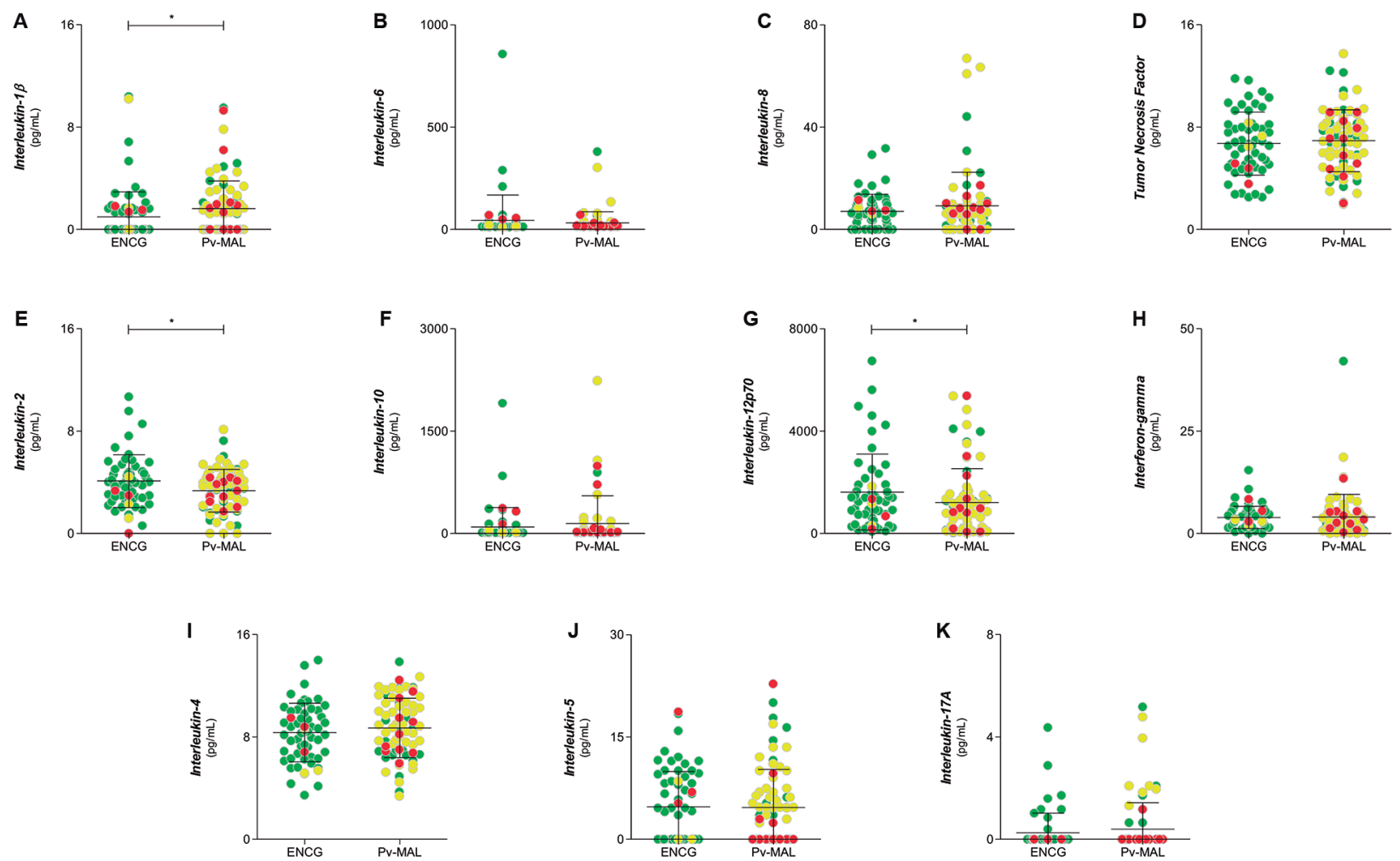

Fig. 4: comparison of serum concentrations of Th1/Th2/Th17 cytokines in endemic control group (ENCG) and Plasmodium vivax malaria (PvMAL). The Th1/Th2/Th17 cytokines was dotted between ENCG and Pv-MAL patients. They were separated in a production order and serum

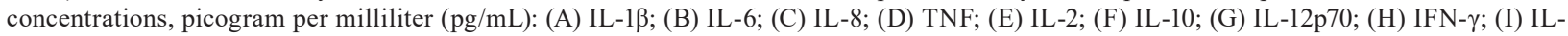
4; (J) IL-5 and (K) IL-17A. The individuals were grouped according in non-thrombocytopenic (green circle), mild (yellow circle) and severe thrombocytopenia (red circle). 
values (Fig. 3B-E). It is also well known that PDW correlates with MPV in normal test subjects, and Fig. 3C-F shows this correlation was lost in Pv-MAL patients.

The serum concentrations of Th1/Th2/Th17 cytokines were compared in the Pv-MAL patients and the endemic control group (Fig. 4). Pv-MAL patients showed a reduction of IL-12p70 and IL-2 levels, while IL-1 $\beta$ was higher in relation to the ENCG (Fig. 4). Other cytokines showed no differences in the comparison between groups.

To assess predictors of thrombocytopenia using cytokine levels of Th1/Th2/Th17 and platelet indices, a multinomial model was used to exclude parasitaemia as a confounding factor (Table). For this, the serum levels of patients with mild parasitaemia were used as a reference group (Table). We observed that the crude odds ratio in the serum concentrations of IL-10, and IL-17A increased individually in response to mild parasitaemia. When Th1/Th2/Th17 cytokines and platelet indices were assessed together, the adjusted odds ratio for the serum concentration of IL-10 and IL-17A also increased in relation to the mild parasitaemia group.

Finally, a linear regression was performed to assess predictors of thrombocytopenia in Pv-MAL patients in relation to the ENCG. Since the PDW index showed negative correlation with platelet counts, we used it as a dependent variable, while the other platelet indices and cytokine levels were considered independent variables (Table). The linear regression showed that the levels of IL-1 $\beta$, IL-2 and IL-12 were associated with altered PDW in Pv-MAL patients, and they may be predictors of the medullar response to thrombocytopenia caused by vivax malaria.

\section{DISCUSSION}

Thrombocytopenia is the most common haematological disorder in patients with malaria and one of the major concerns when accompanied by multiple organ failures. ${ }^{(5,6)}$ In our study, thrombocytopenia was the main manifestation among patients with vivax malaria, though the reduction in platelet count was not associated with parasitaemia. Although some patients presented severe thrombocytopenia, none of them presented bleeding. Very low platelet counts during malaria are considered transient, for those who do not present bleeding, platelet transfusions are unnecessary. ${ }^{(24)}$ Platelet function is compromised in thrombocytopenia caused by malaria, and this is generally evidenced by changes in its parameters. (18) Here, the Pv-MAL patients displayed an increased MPV that may indirectly indicate the presence of mega platelets. ${ }^{(4)}$ A negative correlation between platelet counts and MPV can be observed, but this inverse correlation is not restricted to malaria, since it is usually found in other physiological conditions. ${ }^{(4,18,24)}$ Furthermore, we observed that an inverse correlation between MPV and PDW in the endemic control group was lost in Pv-MAL patients due a trend of increases in PDW and MPV.

The total platelet mass is considered as a product of platelet count X MPV, and this inverse correlation

TABLE

Multivariate analyses of Th1/Th2/Th17 cytokines associated with parasitaemia (binomial) and platelet indices

\begin{tabular}{|c|c|c|c|c|c|c|}
\hline \multicolumn{4}{|c|}{ Logistic regression of Pv-MAL patients in relation to parasitaemia } & \multicolumn{3}{|c|}{$\begin{array}{l}\text { Linear regression predicting PDW between } \\
\text { PV-MAL patients and endemic controls }\end{array}$} \\
\hline $\begin{array}{l}\text { Mild parasitaemia } \\
\text { (Reference) }\end{array}$ & $\begin{array}{l}\text { Crude OR } \\
\text { (CI 95\%) }\end{array}$ & $\begin{array}{l}\text { Adjusted OR } \\
\text { (CI 95\%) }\end{array}$ & $\begin{array}{c}\mathrm{p} \\
\text { (LR-test) }\end{array}$ & $\begin{array}{l}\text { Patients } \\
\text { (Reference) }\end{array}$ & $\begin{array}{l}\text { Estimates } \\
(\text { CI } 95 \%)\end{array}$ & $\mathrm{p}$ value \\
\hline Platelets & $1.00(0.99,1.00)$ & $1.00(0.99,1.01)$ & 0.510 & Platelets & $-0.01(-0.01,0.01)$ & 0.315 \\
\hline MPV & $1.01(0.84,1.21)$ & $1.01(0.75,1.37)$ & 0.947 & MPV & $-0.03(-0.27,0.20)$ & 0.770 \\
\hline PDW & $0.97(0.82,1.16)$ & $0.99(0.81,1.21)$ & 0.93 & PDW & $1.44(0.24,2.63)$ & 0.019 \\
\hline IL-1 $\beta$ & $1.02(0.99,1.06)$ & $1.03(0.99,1.07)$ & 0.100 & IL-1 $\beta$ & $-0.03(-0.05,-0.01)$ & 0.047 \\
\hline IL-6 & $1.00(0.99,1.00)$ & $1.00(0.99,1.0001)$ & 0.123 & IL-6 & $-0.01(-0.01,0.01)$ & 0.446 \\
\hline IL-8 & $1.00(0.99,1.01)$ & $0.99(0.98,1.00)$ & 0.197 & IL-8 & $-0.01(-0.01,0.01)$ & 0.801 \\
\hline TNF & $1.00(0.99,1.01)$ & $1.01(0.99,1.03)$ & 0.312 & TNF & $0.01(-0.01,0.01)$ & 0.371 \\
\hline IL-2 & $1.00(0.98,1.01)$ & $0.99(0.96,1.01)$ & 0.301 & IL-2 & $-0.02(-0.04,-0.01)$ & 0.030 \\
\hline IL-10 & $1.00(1.00,1.00)$ & $1.00(1.00,1.00)$ & 0.029 & IL-10 & $-0.01(-0.01,0.01)$ & 0.573 \\
\hline IL-12p70 & $1.00(0.99,1.00)$ & $1(0.99,1.00)$ & 0.692 & IL-12p70 & $-0.01(-0.01,-0.01)$ & 0.038 \\
\hline IFN- $\gamma$ & $1.00(0.99,1.00)$ & $0.99(0.99,1.01)$ & 0.900 & IFN- $\gamma$ & $-0.01(-0.01,0.01)$ & 0.413 \\
\hline IL-4 & $0.99(0.99,1.00)$ & $0.99(0.98,1.00)$ & 0.122 & IL-4 & $0.01(-0.01,0.01)$ & 0.833 \\
\hline IL-5 & $1.00(0.96,1.04)$ & $0.99(0.94,1.04)$ & 0.773 & IL-5 & $0.04(-0.01,0.08)$ & 0.052 \\
\hline IL-17A & $1.02(1.00,1.05)$ & $1.03(1.00,1.07)$ & 0.043 & IL-17A & $-0.01(-0.03,0.02)$ & 0.712 \\
\hline
\end{tabular}

CI: confidence interval; MPV: mean platelet volume; OR: odds ratio; PDW: platelet distribution width; Pv-MAL: Plasmodium vivax malaria. 
may be closely regulated. ${ }^{(23)}$ Cytokine levels in malaria have dual effects in the pathogenesis of, and protection against, malaria. ${ }^{(25)}$ The cytokine imbalance in thrombocytopenia caused by vivax malaria has been reported by several studies without a consensus about which cytokines could be associated to thrombocytopenia. $(4,13,20,26,27)$ In this study, we assessed immune responses in relation to other parameters as well as platelet counts. Of all the cytokines, only IL-1 $\beta$, IL-12 and IL-2 differed in Pv-MAL patients, and were associated with increases in the PDW values. Thus, the association of PDW with these differences may be considered a reduction of Th1 responses and thus the main mechanism which induces the elevation of MPV and PDW, in accordance with the findings of other authors. ${ }^{(18)}$

Furthermore, parasitaemia was associated with higher levels of IL-10 and a slight increase in the serum concentration of IL17A. Increased levels of IL-10 have already been seen in patients with higher parasitaemia and patients with recurrent malaria. ${ }^{(28,29)}$ Here, this imbalance in IL-10 and IL17A seems to be a shift from a pro-inflammatory response to an anti-inflammatory response, which explains the low frequencies of severe malarial disease among individuals living in endemic areas. ${ }^{(29)}$

This study had several limitations. The sample size of patients was small and PCR for malaria diagnoses was not performed in all of those enrolled in this study. Nor did we evaluate antimicrobial failure. The population of the municipality of Coari is very well-informed about malaria and knows about the need to diagnose malaria at the onset of the disease, hence, this may explain why all the patients had uncomplicated malaria. Another limitation to the study was the semi-quantification of parasites which is carried out in the Brazilian Amazon, and which made it impossible to assess further correlations, as also observed by a few other studies. ${ }^{(18,30)} \mathrm{A}$ further limitation to this study was that documentation of mega platelets was not done at the time of blood collection, however, the aim of the study was to assess changes in the immune response associated with thrombocytopenia. It is likely that the changes in MPV and PDW observed here may be due the release of the larger platelets also called as mega platelets or giant platelets. ${ }^{(4,19)}$ These mega platelets may be detected indirectly by the increase in platelet volume in thrombocytopenia caused by malaria. The greater amount of $\alpha$-granules and dense granules enhance the mega platelets' ability of to adhere and aggregate with small platelets, as observed in peripheral blood smears of children infected with $P$. falciparum malaria. ${ }^{(19)}$ These increases provide equal or similar primary haemostasis and may be the reason why bleeding episodes are rare in acute malaria infection, despite the thrombocytopenia. ${ }^{(4,19)}$ Thus, the reason for our assumption is that megakaryocytic lineage is apparently preserved in bone marrow and able to release mega platelets in the blood stream. ${ }^{(4,13,18)}$ Our data encourage studies that assess the release of mega platelets in thrombocytopenia caused by $P$. vivax infections.

In conclusion, the increase in MPV indirectly indicated the presence of mega platelets. The loss of its correlation with PDW in Pv-MAL patients may also be a consequence of the release of mega platelets. The reduction of IL-2 and IL-12 associated with PDW may be consistent with regulation of immune response which is associated with appearance of mega platelets in thrombocytopenia caused by vivax malaria.

\section{ACKNOWLEDGEMENTS}

To the collaboration of the local microscopists from Instituto de Medicina Tropical de Coari (IMTC) and laboratory support of the Laboratório de Marcadores Celulares (HEMOAM). The authors also thank the Program for Technological Development in Tools for Health (PDTIS/FIOCRUZ) for the use of its facilities.

\section{AUTHORS' CONTRIBUTION}

AGC, AM, YC, LB, MPM and PAN were responsible for the data collection from medical records; $\mathrm{AB}$ and WMM performed the statistical analysis; ACG, LB, ATC, OAMF, LRVA and AM participated in study design; ACG, AM, OAMF and PAN wrote the first draft of the manuscript; AGC, OAMF, RR, FTMC, MVGL and PAN elaborated the final version of manuscript. All authors read and approved the final manuscript. The authors declare that the funders had no role in study design, data collection and analysis, decision to publish, or preparation of the manuscript.

\section{REFERENCES}

1. Patel U, Gandhi G, Friedman S, Niranjan S. Thrombocytopenia in malaria. J Natl Med Assoc. 2004; 96(9): 1212-14.

2. Wickramasinghe SNN, Abdalla SHH. Blood and bone marrow changes in malaria. Baillieres Best Pract Res Clin Haematol. 2000; 13(2): 277-99.

3. Saravu K, Docherla M, Vasudev A, Shastry BA. Thrombocytopenia in vivax and falciparum malaria: an observational study of 131 patients in Karnataka, India. Ann Trop Med Parasitol. 2011; 105(8): 593-8.

4. Lacerda MVG, Mourão MPG, Coelho HCC, Santos JB. Thrombocytopenia in malaria: who cares? Mem Inst Oswaldo Cruz. 2011; 106(Suppl. I): 52-63.

5. Hanson J, Phu NH, Hasan MU, Charunwatthana P, Plewes K, Maude RJ, et al. The clinical implications of thrombocytopenia in adults with severe falciparum malaria: a retrospective analysis. BMC Med. 2015; 13: 97.

6. Naing C, Whittaker MA. Severe thrombocytopaenia in patients with vivax malaria compared to falciparum malaria: a systematic review and meta-analysis. Infect Dis Poverty. 2018; 7(1): 10.

7. Rahimi BA, Thakkinstian A, White NJ, Sirivichayakul C, Dondorp AM, Chokejindachai W. Severe vivax malaria: a systematic review and meta-analysis of clinical studies since 1900. Malar J. 2014; 13: 481.

8. Tanwar GS, Khatri PC, Chahar CK, Sengar GS, Kochar A, Tanwar G, et al. Thrombocytopenia in Childhood malaria with special reference to $P$. vivax monoinfection: a study from Bikaner (Northwestern India). Platelets. 2012; 23(3): 211-6.

9. Cox D, McConkey S. The role of platelets in the pathogenesis of cerebral malaria. Cell Mol Life Sci. 2010; 67(4): 557-68.

10. Karanikas G, Zedwitz-Liebenstein K, Eidherr H, Schuetz M, Sauerman R, Dudczak R, et al. Platelet kinetics and scintigraphic imaging in thrombocytopenic malaria patients. Thromb Haemost. 2004; 91(3): 553-7. 
11. Grau GE, Mackenzie CD, Carr RA, Redard M, Pizzolato G, Allasia $\mathrm{C}$, et al. Platelet accumulation in brain microvessels in fatal pediatric cerebral malaria. J Infect Dis. 2003; 187(3): 461-6.

12. Mast Q, Groot PG, Van Heerde WL, Roestenberg M, Van Velzen JF, Verbruggen B, et al. Thrombocytopenia in early malaria is associated with GP1b shedding in absence of systemic platelet activation and consumptive coagulopathy. Br J Haematol. 2010; 151(5): 495-503.

13. Coelho HCC, Lopes SCP, Pimentel JPD, Nogueira PA, Costa FTM, Siqueira AM, et al. Thrombocytopenia in Plasmodium vivax malaria is related to platelets phagocytosis. PLoS One. 2013; 8(5): e63410.

14. Geddis AE. Megakaryopoiesis. Semin Hematol. 2010; 47(3): 212-9.

15. Müller-Newen G, Stope MB, Kraus T, Ziegler P. Development of platelets during steady state and inflammation. J Leukoc Biol. 2017; 101(5): 1109-17.

16. Behrens K, Alexander WS. Cytokine control of megakaryopoiesis. Growth Factors. 2018; 36(3-4): 89-103.

17. Mast Q, Groot E, Asih PB, Syafruddin D, Oosting M, Sebastian $\mathrm{S}$, et al. ADAMTS13 deficiency with elevated levels of ultra-large and active Von Willebrand factor in P. falciparum and $P$. vivax malaria. Am J Trop Med Hyg. 2009; 80(3): 492-8.

18. Leal-Santos FA, Silva SBR, Crepaldi NP, Nery AF, Martin TOG, Alves-Junior ER, et al. Altered platelet indices as potential markers of severe and complicated malaria caused by Plasmodium vivax: a cross-sectional descriptive study. Malar J. 2013; 12: 462.

19. Maina RN, Walsh D, Gaddy D, Hongo G, Waitumbi J, Otieno L, et al. Impact of Plasmodium falciparum infection on haematological parameters in children living in Western Kenya. Malar J. 2010; 9(Suppl. 3): 54.

20. Park JW, Park SH, Yeom JS, Huh AJ, Cho YK, Ahn JY, et al. Serum cytokine profiles in patients with Plasmodium vivax malaria: a comparison between those who presented with and without thrombocytopenia. Ann Trop Med Parasitol. 2003; 97(4): 339-44.
21. MS - Ministério da Saúde do Brasil [homepage on the Internet]. Sistema de informação da vigilância epidemiológica - Malária (SIVEP-Malária). [updated 2015 June 15; cited 2020 February 8]. Brasília. Available from: www.saude.gov.br/sivep_malaria.

22. Lampah DA, Yeo TW, Malloy M, Kenangalem E, Douglas NM, Ronaldo D, et al. Severe malarial thrombocytopenia: a risk factor for mortality in Papua, Indonesia. J Infect Dis. 2015; 211(4): 623-34.

23. Lippi G, Favaloro EJ. Laboratory hemostasis: milestones in clinical chemistry and laboratory medicine. Clin Chem Lab Med. 2013; 51(1): 91-7.

24. Gupta P, Guddattu V, Saravu K. Characterization of platelet count and platelet indices and their potential role to predict severity in malaria. Pathog Glob Health. 2019; 113(2): 86-93.

25. Andrade BB, Reis-Filho A, Souza-Neto SM, Clarêncio J, Camargo LMA, Barral A, et al. Severe Plasmodium vivax malaria exhibits marked inflammatory imbalance. Malar J. 2010; 9: 13.

26. Punnath K, Dayanand KK, Chandrashekar VN, Achur RN, Kakkilaya SB, Ghosh SK, et al. Association between inflammatory cytokine levels and thrombocytopenia during Plasmodium falciparum and $P$. vivax infections in south-western coastal region of India. Malar Res Treat. 2019; 2019: 4296523.

27. Raza A, Khan MS, Ghanchi NK, Raheem A, Beg MA. Tumour necrosis factor, interleukin- 6 and interleukin-10 are possibly involved in Plasmodium vivax-associated thrombocytopaenia in southern Pakistani population. Malar J. 2014; 13: 323.

28. Costa AG, Antonelli LRV, Costa PAC, Pimentel JPD, Garcia NP, Tarragô AM, et al. The robust and modulated biomarker network elicited by the Plasmodium vivax infection is mainly mediated by the IL-6/IL-10 axis and is associated with the parasite load. J Immunol Res. 2014; 2014: 318250.

29. Chaves YO, Costa AG, Pereira MLM, Lacerda MVG, CoelhoDos-Reis JG, Martins-Filho OA, et al. Immune response pattern in recurrent Plasmodium vivax malaria. Malar J. 2016; 15(1): 445.

30. Rubio E, Alejo-Cancho I, Aylagas C, Camprubí D, Ferré R, Albarracín $\mathrm{MR}$, et al. Diagnostic value of platelet and leukocyte counts in the differential diagnosis of fever in the returning traveler. Am J Trop Med Hyg. 2019; 100(2): 470-5. 